\title{
From research to policy: the role of health research institution in social development in Africa ${ }^{¥}$
}

\author{
DAVID H. MWAKYUSA* \\ Ministry of Health and Social Welfare, P.O. Box 9083, Dar es Salaam, Tanzania
}

I would like to start my address by thanking the Conference Organizing Committee for inviting me, for the second time in a row, to officiate the opening the twenty - second Annual Joint Scientific Conference of the National Institute for Medical Research. I am delighted to note that this annual conference activity of NIMR has been an internationally recognized forum for scientific exchange.

On behalf of the Government of the United Republic of Tanzania, and on my own behalf, I wish to extend to all of you a warm and hearty welcome. I am particularly pleased to learn that amongst you are participants from other countries spanning several continents. You are very welcome to Tanzania and in particular to this beautiful city of Arusha. Please feel at home and try to apportion some of your time to experience Tanzanian hospitality.

I would like at the outset to recognize the presence at this conference of the Canadian High Commissioner to Tanzania, Dr. Andre McAllister. From the time he took up his position here in Tanzania, Dr. McAllister has all along shown keen interest in health and health research development in Tanzania and his country's contribution to the development of the health sector in Tanzania is enormous.

I am further informed that the Honourable High Commissioner has contributed substantial financial support to the $21^{\text {st }}$ Annual Scientific Conference last year as well as to this one. We appreciate and treasure your contributions and support in whatever form.

I would like to emphasize the importance of research for social and economical development. The global picture shows clearly that countries which have had strong culture of research and which have invested in developing quality research capacities are the ones enjoying a high social status and have better control of the global economics. Research has contributed better diagnostic and disease management tools, appropriate and adequate disease prevention and control strategies, and research continues to guide the conduct of interventions for greater input.

To be meaningful, research should be geared to addressing people's concerns and problems, to find solutions to questions like why, when, how and what. In academic institutions we come across a lot of research that individuals conduct in order to fulfil requirements for acquisition of a diploma. After that the research findings do not attract any application and are therefore stacked away on the shelf. Such research has no place in the social advancement of our country, and indeed of any country. Research should be people centred, hence the appropriateness and relevance of the theme of this year's conference: "From research to policy: the role of health research institution in social development in Africa"

As stated earlier, research must be designed in such a way that its potential findings will bring about change. It is obvious that the mere generation of new knowledge emanating from contemporary research does not guarantee its effective application. We need to put in place mechanisms which will ensure that this new information is timely transmitted to individuals and communities for them to make informed decisions and incorporation of the findings in national policies.

I would like to invite you to run with me through four categories of problems which are major stumbling blocks in bridging the gap between research and policy formulation: Category one relates to ignorance of the value and potential contribution of the new knowledge or tools towards solving the problems at hand. New knowledge may be available and yet inaccessible to the intended. The second category relates to the natural tendency or predisposition of the individual, community or policy maker to stick to the old way of doing things while being

\footnotetext{
${ }^{\ddagger}$ Opening address during the $22^{\text {nd }}$ Annual Joint Scientific Conference of the National Institute for Medical Research, Arusha, Tanzania, $7^{\text {h }}$ March, 2007

${ }^{*}$ Minister for Health and Social Welfare, United Republic of Tanzania
} 
full aware of the existence of the new knowledge and its potential benefits. This is mainly caused by the inertia of holding onto the old familiar ways, and a natural resistance to change. The third category relates to affordability of adopting the new knowledge, a factor closely linked to poverty. Individuals or the system may be unable to afford health products or services even when they are excellent and could offer great benefits. Fourth and last category is the occasional lack of guidance by scientists on how to implement a proposed policy or intervention.

I would like to take this opportunity to illustrate some of these difficulties by giving a couple of practical examples. In 1998 Tanzanian Scientists presented for the first time date showing alarming levels of the malaria parasite resistance against chloroquine, the anti-malarial drug which was until then, the cheapest, safest and effective. The information was presented as a paper in a research forum, and alarming as it was, it still left a lot of questions unanswered for a policy decision to be made then. The type of questions included whether the resistance as geographically localized or widespread in the country, whether there was a suitable alternative choice of substitute drugs, how the alternative choice compared in effectiveness with chloroquine and finally and equally important questions was, whether we could afford to switch over to the alternative option and if the change, once made, would be sustainable.

After considerable discussions, the research community agreed to go back and search for answers to at least some of these questions. It was only in 2001 after the questions had been answered adequately that the policy makers were satisfied to implement the prescribed changes. It should be noted, however, that even after the policy pronouncement, the public was not very willing or ready to effect the change and it took some time to win their support. In this respect, any policy change requires your support as scientists to inform better and correctly the public to enhance compliance.

Another example is the story of insecticide treated nets (ITNs). I am informed that there was initial resistance from the communities, especially communities living along the coastal belt, to accept to sleep under a white mosquito net. It was not evident why and it took some time to understand the local beliefs and practices associated with funeral services where a white shawl similar to the net is used to cover the dead body. For these communities, change of colour was the immediate solution, thanks to the work of social scientists whose probes led to this discovery. This is an example of a new useful tool whose use was impeded by the local beliefs.

Yet another example, while wondering why the usage rate of ITNs in Kagera was only 3\% of the population, the reason was traced back to a television advert. In this advert a child was sleeping fitfully under an ITN; the moment a mosquito touched the net, it dropped dead. The parents were not sure if it was safe to leave their children sleeping all night under a net that was so deadly, they were not sure whether the poison in the net could distinguish a mosquito from a baby. The commercial was dropped and the compliance has improved.

I bet the scientists gathered in this room can come up with many more examples where tools or new knowledge have not been fully utilized for various reasons, and hence individuals and populations are not benefiting as they might otherwise have done. The lessons to learn here are that, there is great need for packaging and if necessary, repacking the information in a form that is understandable to the targeted user. This packaging should include language simplification and providing the rationale or making the user appreciate the benefits and what they would miss by not making it their choice. It also means greater involvement in dialogue between the researchers, policy makers and users so that they can understand each other's way of thinking. The proposed changes, needless to say are not generic; there is need to understand the socio-cultural context within which the change is expected to take place.

I am delighted to hear that the National Institute for Medical Research (NIMR) is playing a pivotal role in spearheading the introduction of a systematic mechanism of bridging the gap that exists between research and policy. I am also informed that a strong initiative under the name of REACH Policy Initiatives has already been put in place and I am aware that there has been established already, with the East African Community a Health Desk.

This initiative, I have been made to understand, is intended to become a permanent activity of the East African Health Research 
Commission that is in the process of being formed. For all this, I wish to congratulate all those who have taken the foresighted initiative to put such a process in place, and also for the considerable achievements made so far. I know that NIMR will be the Country Node for Tanzania and that NIMR in close collaboration with my Ministry has already been working on the establishment of the National Health Research Information Centre in Dar es Salaam where the research to policy analysis and dissemination will be based. I hope that this Node will also endeavour to establish smaller nodes at regional and district levels to ensure that appropriate consumption of research information reaches where the information is actually put to practice and where real action in utilizing the newly generated information takes place.

I would also like to emphasize that; we need to strive for maximum use of the media to enhance correct information dissemination. I urge you not to stop at satisfying only policy makers but to first and foremost direct it to the public. It is the individuals and communities whose change of attitude will make a difference in adopting health promoting behavioural practice. If we succeed in empowering the individual and communities through proper and effective health information dissemination, we will be assured of making a difference in adopting informed policy decisions, which in this case, will now come by demand of the well informed and subsequently, converted individuals and communities.

The Government of the United Republic of Tanzania through my Ministry has long recognized the contribution of health research towards development. This is why the National Institute for Medical Research was established in the first place, and my Ministry is investing not only in maintaining the current establishment, but also in building further its capacities to conduct quality research and its proper translation to useful end product capable of providing solutions to our health problems. The emphasis is on research whose results, if applied appropriately will bring forth the required rapid improvement in the health status of Tanzanians.

While promoting and strengthening research endeavours, we must also set in motion adequate mechanisms for monitoring and evaluation of interventions. Monitoring and evaluation are absolutely essential for maintaining good management, ensuring responsibility accountability and transparency not only of industrial and commercial enterprises but also of health research investments.

The Tanzanian tax payer has the right to gain easy access to research results, in the manner and form that he can make best use of. It is that information which is a crucial input for Tanzania's social economic transformation. The urgency of proper translation of research findings into meaningful policy and practice cannot be over-emphasized.

At this juncture, I cannot over-emphasize the need to create a strong forum for the dissemination of information on research findings that may guide appropriate and prompt action. Very recent examples of the outbreaks of SARS, Avian Influenza and Rift Valley Fever virus are just a few. Our research scientists must keep abreast, not only with traditional research work, but also deal with these urgent and emerging, and often deadly diseases. My Ministry is ready and willing to assist health researchers in bridging this information gap by all means within our reach. Similarly all institutions conducting research in Tanzania should strive to establish mechanisms for disseminating own research findings and communicate effectively with their clientele, including fellow researchers and the public education sector. This must be a continuous sustained process.

Researchers must know that if they cannot efficiently and effectively deliver to the stakeholders their research findings, then they have failed to accomplish their mandate. In fact, un-disseminated research findings do not only become useless but also make for multiple losses to the nation; a waste of precious time, a loss of funds invested in the work and human power, a loss of productivity and a loss to people's welfare.

There is no doubt that you have a very busy schedule ahead of you. But as the nursery rhyme goes, "All work and no ply makes Jack a dull boy" I believe you will not find Arusha dull. Please take time off to visit some of the attractive spots that Arusha offers. You will be interested to learn that with its invigorating climate Arusha is exactly midway between the Cape and Cairo. Depending on your particular tastes, there are various attractions with a short drive of this Municipality. 
Foremost, there is Mount Kilimanjaro, which is clearly the highest mountain in Africa. You may wish to take a leisure drive around it, or if you are a seasoned mountaineer, there lies yet another challenge, and another record to be set. In case you are a naturalist, there are a variety of sites waiting to be explored. Among these are the Olduvai Gorge, the real cradle of humankind. The Serengeti National Park, which by any measure is among the best in the world; the Ngorongoro Crater dubbed by some as the Garden of Eden, and the Lake Manyara National Park with its very unique tree climbing lions, and close nearby here are the Momella, Ngurdoto, Arusha and Tarangire National Parks; and all these are at your disposal to explore and enjoy! Please spare some time to do justice to these marvels of nature. 


\section{Information and instructions for Authors}

\section{General Information}

Tanzania Health Research Bulletin is a peer reviewed journal which targets scientists, policy and decision makers, as well as the public. The Editorial Board welcomes contributions in the form of original research reports, review articles, short communications, commentaries or letters to the editor on health research, public health, and policy and planning.

Manuscripts should be prepared in accordance with the fifth edition of the "Uniform Requirements for Manuscripts submitted to Biomedical Journals" established by the Vancouver Group (International Committee of Medical Journal Editors, ICMJE). The complete document, updated October 2001, is available at http:/ / www.icmje.org/index.html

Manuscripts are considered with the understanding that they have neither been published previously nor are under consideration by another publisher.

\section{Preparing a manuscript}

The manuscript should be organized as follows:

Title. The title should be short and to the point; omit phrases such as "Investigation on...' "Preliminary report of ...'; numbered series articles followed by a subtitle are not allowed. Taxonomic affiliation and authority should be given in the abstract, or in the key words, but not in the Title.

Addresses. Full addresses should appear under the names of the author(s); in addition, the title page should contain the name and address of the person to whom the proofs should be sent. If the author's address has changed, his full present address should also appear on the title page.

Key words. A short list of terms suitable for indexing should be included.

\section{Body of manuscript}

a) Abstract (short and concise): this should contain the full scientific name

b) Introduction: this part should be directed to the present investigation; avoid giving a review on general aspects of the topic; number of citation should be limited.

c) Materials and methods: all information must be given so that it is quite clear how the experiments were done: any interested colleague should be able to verify the experiments from this information. However, details about already commonly used methods and materials must be omitted. Information about the origin of the source of materials is necessary.

d) Results: the results are usually presented in tables and figures, and the text must provide a clear explanation of this information. Tables and figures should only be used for substantial amounts of data, otherwise the information should be inserted in the text. Avoid repeating data from Tables and Figures in the text as much as possible.

e) Discussion: additional citations may be given here, and the findings discussed in relation to what is already known. Scientific as well as practical implications may be mentioned. Within space limitations the author has some freedom to express his own opinion, even if editors or referees differ with him.

f) Tables: Data in tables and figures should not be duplicated. They should be numbered consecutively in Arabic numerals, and bear descriptive headings. Each is to be presented individually on separate sheets. If many tables cover similar results, try to combine them. Statistical significances should be indicated with small letters after the data with a note of explanation under the table. Remember that in English data with decimals are written 7.5. Usage of more decimals than necessary should be avoided.

g) Figures: Drawings should also be restricted and combining drawing under one caption must be considered. Figure legends should be grouped together on a separate sheet. The figures should be identified by number and name of author.

h) References: For citations in the text: use name and year system; e.g. (Magesa, 1998); for two 
authors, use the ampersand (\&); e.g. (Mboera \& Kitua, 2001); for more than two authors, use et al., e.g. (Mboera et al., 1999). If referring to a personal communication use initials e.g. (A.Y. Kitua, per comm.). For unpublished results, use unpubl., e.g. (Ijumba, unpubl). The later two categories, however, cannot be put in the reference list.

For citations in the reference section: list each reference alphabetically on the first author's last name; if a single author has more than one contribution, list each chronologically; in cases of more than one author, list each reference alphabetically on $2^{\text {nd }}, 3^{\text {rd }}$, etc. author's names. Publications in preparation or submitted can only appear in the reference list if they have been accepted for publication, mentioning year of publication and volume of the journal with the reference note 'in press' added. Journal titles should be given in full.

When a scientific name of an animal or a plant is used for the first time in an article, it should be stated in full. In subsequent citations, the genus name may be abbreviated to its first letter followed by a period.

i) Units, symbols and abbreviations. Numerals are always used with abbreviated units (e.g. $3 \mathrm{~mm}^{2}$ not three $\mathrm{mm}^{2}$ ). In all other cases, whole numbers above 10, fractions and decimals should be expressed as numerals, and whole numbers between 1 and 10 are to be spelled out in full. All units are to conform to the standards of the International System (S.I)

\section{Short communications}

Contributors are requested to submit short manuscripts (up to three pages in final print) as 'short communications'. A short communication may contain an abstract, and can be organized either along the lines of a regular manuscript, or without subdivisions. Authors may also consider combining the results and discussion sections.

\section{Clinical studies}

The Bulletin supports the registration of trials as an important initiative to improve the reporting of clinical studies. Trial registers that currently meet all of the International Committee of Medical Journal Editors (ICMJE) and World Health Organization (WHO) requirements can be found at http: / / www.icmje.org/faq.pdf

\section{Submitting a manuscript}

The manuscript should be submitted preferably by email to:

The Editor, Tanzania Health Research Bulletin, National Institute for Medical Research, P.O. Box 9653, Dar es Salaam, Tanzania; Fax: +255 22 2121360.Email: thrb@nimr.or.tz or lmboera@nimr. or.tz 